\title{
Recognizing Reverberant Speech Based on Amplitude and Frequency Modulation
}

\author{
Yotaro KUBO $^{\dagger \text { a) }}$, Student Member, Shigeki OKAWA ${ }^{\dagger \dagger}$, Member, Akira KUREMATSU $^{\dagger}$, \\ and Katsuhiko SHIRAI ${ }^{\dagger}$, Fellows
}

SUMMARY We have attempted to recognize reverberant speech using a novel speech recognition system that depends on not only the spectral envelope and amplitude modulation but also frequency modulation. Most of the features used by modern speech recognition systems, such as MFCC, PLP, and TRAPS, are derived from the energy envelopes of narrowband signals by discarding the information in the carrier signals. However, some experiments show that apart from the spectral/time envelope and its modulation, the information on the zero-crossing points of the carrier signals also plays a significant role in human speech recognition. In realistic environments, a feature that depends on the limited properties of the signal may easily be corrupted. In order to utilize an automatic speech recognizer in an unknown environment, using the information obtained from other signal properties and combining them is important to minimize the effects of the environment. In this paper, we propose a method to analyze carrier signals that are discarded in most of the speech recognition systems. Our system consists of two nonlinear discriminant analyzers that use multilayer perceptrons. One of the nonlinear discriminant analyzers is HATS, which can capture the amplitude modulation of narrowband signals efficiently. The other nonlinear discriminant analyzer is a pseudo-instantaneous frequency analyzer proposed in this paper. This analyzer can capture the frequency modulation of narrowband signals efficiently. The combination of these two analyzers is performed by the method based on the entropy of the feature introduced by Okawa et al. In this paper, in Sect. 2, we first introduce pseudo-instantaneous frequencies to capture a property of the carrier signal. The previous AM analysis method are described in Sect. 3. The proposed system is described in Sect.4. The experimental setup is presented in Sect. 5, and the results are discussed in Sect. 6. We evaluate the performance of the proposed method by continuous digit recognition of reverberant speech. The proposed system exhibits considerable improvement with regard to the MFCC feature extraction system.

key words: speech recognition, temporal feature, tandem approach, multistream combination, reverberant speech

\section{Introduction}

Most of the features used by modern speech recognition systems, such as Mel-frequency cepstral coefficients (MFCC), perceptual linear prediction (PLP) [1], and temporal patterns (TRAPS) [2], are derived from the energy envelopes of narrowband signals by discarding the information in the carrier signals. However, some experiments show that apart from the spectral/time envelope and its modulation, the information on the zero-crossing points of the carrier signals also plays a significant role in human speech recognition [3].

Manuscript received June 28, 2007.

Manuscript revised September 12, 2007.

${ }^{\dagger}$ The authors are with the School of Science and Engineering, Waseda University, Tokyo, 169-8555 Japan.

${ }^{+\dagger}$ The author is with the Chiba Institute of Technology, Narashino-shi, 275-0061 Japan.

a) E-mail: yotaro@shirai.cs.waseda.ac.jp

DOI: 10.1093/ietisy/e91-d.3.448
In realistic environments, a feature that depends on a limited number of properties of the signal may easily be corrupted. In order to utilize an automatic speech recognizer in an unknown environment, using the information obtained from other signal properties and combining them is important to minimize the effects of the environment [4]. In this paper, we propose a method to analyze carrier signals that are discarded in other speech recognition systems.

A realistic environment that involves difficulties with regard to speech recognition is a reverberant sound field. Reverberant speech can be produced by the convolution of an impulse response; hence, reverb can be handled as multiplicative noise that can be theoretically suppressed by using cepstral mean normalization (CMS). However, CMS is not practically efficient because most impulse responses in reverberant environments have durations that are greater than the analysis frame length. The effects of such long impulse responses can be considered as context-dependent additive noise.

Relative spectra (RASTA) [5] and TRAPS [2] are robust feature analysis methods for reverberant speech; these methods use the amplitude modulation (AM) of narrowband speech signals. However, these techniques also have their limitations. For example, it is known that reverberant environments act as low-pass filters of envelope signals that cause the degradation of AM features [6]. Therefore, it is important to combine the relevant aspects that are robust to reverberant environments.

In order to overcome such limitations, the method which uses the average instantaeous frequency (AIF) are proposed by Wang et al. [7]. In this method, the AIFs are considered for capturing the state of the carrier signal. However, we consider that temporal aspects are more important for carrier analysis.

Previous methods for capturing carrier signals are based on stochastic quantities such as the average frequency and modulation percentage of temporal segments [7], [8]. These methods eliminate temporal structures in an instantaneous frequency. In order to use the temporal structures of the instantaneous frequency, we propose more straightforward feature extraction method.

The importance of temporal aspects of speech recognition has been discussed along with the importance of AM information [4], [9]-[11]. An investigation on the importance of frequency modulation (FM) is also important in order to achieve robust speech recognition. 
In this paper, we propose a method to analyze FM signals that are discarded in other speech recognition systems.

Our system consists of two nonlinear discriminant analyzers that use multilayer perceptrons (MLPs). One of the nonlinear discriminant analyzers is HATS introduced by Chen et al. which can capture the AM of narrowband signals efficiently [11]-[13]. The other nonlinear discriminant analyzer is a pseudo-instantaneous frequency analyzer proposed in this paper. This analyzer can capture the FM of narrowband signals efficiently. The combination of these two analyzers is performed by the method based on the entropy of the feature introduced by Okawa et al. [14].

In this paper, in Sect. 2, we first introduce pseudoinstantaneous frequencies for capturing a property of the carrier signal. The previous AM analysis method are described in Sect.3. The proposed system is described in Sect. 4. The experimental setup is presented in Sect.5, and the results are discussed in Sect. 6.

\section{Pseudo-Instantaneous Frequency (PIF)}

One of the most popular carrier analysis methods involves the extraction of the instantaneous phase using an analytic signal derived by using Hilbert transforms. The instantaneous frequency (IF), which is the derivative of the instantaneous phase, is often used for understanding the FM of signals.

The IF $\omega(n)$ of the signal $x(n)$ is defined by

$$
\begin{aligned}
& x^{+}(n)=x(n)+j \hat{x}(n), \\
& \phi(n)=\arg x^{+}(n), \\
& \omega(n)=\frac{d}{d n} \phi(n) .
\end{aligned}
$$

Here, $\hat{x}(n)$ is the Hilbert transform of $x(n) ; x^{+}(n)$, the analytic signal of $x(n)$; and $\phi(n)$, the instantaneous phase (IP) of $x(n)$.

There is a practical problem in the determination of the
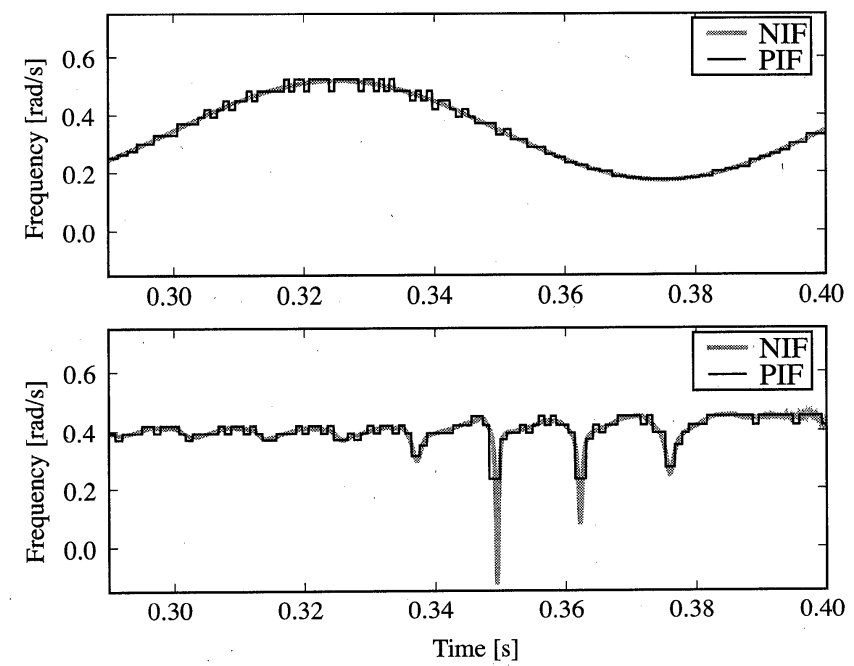

Fig. 1 IF obtained by the numerical approach (NIF) [15] and the PIF of a single sinusoid with FM (top) and a narrowband speech signal (bottom).
IF: IF cannot be defined when $x^{+}(n)=0$.

In order to eschew this behavior, we defined a pseudo-instantaneous frequency (PIF) that respects the zerocrossing points of the carrier signal. The PIF is obtained by performing following steps:

1. Measure the time interval $D(n)$ between the preceding and the following zero-crossing points of each sample.

2. The PIF at time $n$ is defined by $\pi / D(n)$.

The PIF represents the instantaneous density of the zero-crossing points; the instantaneous density depends on the IF and has strong correlation with the IF (Fig. 1).

\section{Hidden Activation TRAPS (HATS)}

In this section, we describe about AM analysis method introduced by Chen $e$ t al. that can emphasize significant modulation components using MLPs. Our objective is applying this method to PIF signals.

Figure 2 shows the block diagram of HATS.

\subsection{AM-Emphasis Using MLP-OL}

First, HATS separates the input signal using Bark filter bank [1]. Then, the output of the filter bank are processed by MLP-OL.

MLP-OLs $^{\dagger}$ are used to extract the significant modulation components from an envelope. MLP-OL is the general MLP classifier during the training phase (Fig. 3).

The input signal $x_{i}$ of the $i^{\text {th }}$ neuron in the input layer of the MLP-OL at time $n$ is defined by

$$
x_{i}=E_{b}\left(n+i-\frac{L+1}{2}\right) .
$$

Here, $L$ is the number of dimensions of the input vector (must be odd) and $E_{b}(n)$ is the energy of the output of the $b^{\text {th }}$ channel of the filter bank at time $n$. Typically, sampling

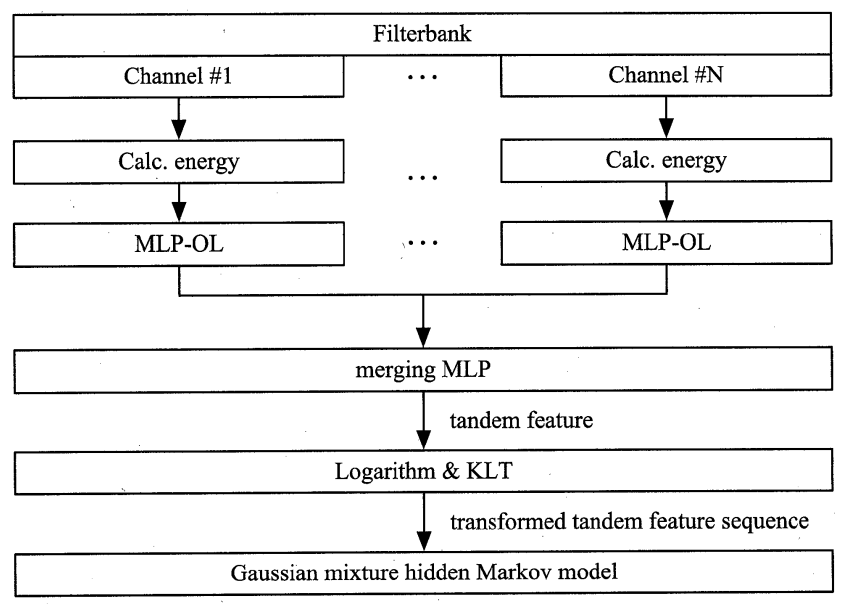

Fig. 2 Block diagram of HATS.

${ }^{\dagger}$ MLP-OL stands for MLP minus output layer. 
rate of $E_{b}$ is set to $100 \mathrm{~Hz}$, and $L$ is set to 51 .

The training session is formulated by the following equations.

$$
\begin{aligned}
& M\left(x, F, k_{1}\right)=s\left(F x+k_{1}\right) . \\
& \hat{F}, \hat{W}, \hat{k_{1}}, \hat{k_{2}}=\underset{F, W, k_{1}, k_{2}}{\operatorname{argmin}}\left|\hat{y}-s\left(W \cdot M\left(x, F, k_{1}\right)+k_{2}\right)\right|^{2} .
\end{aligned}
$$

Here, $s(x)$ is a function that applies sigmoid function to each element of vector $x ; \hat{y}$, the teaching signal; $F$, the weight matrix between the input layer and the hidden layer; $k_{1}$, the bias term of the hidden layer; $W$, the weight matrix between the hidden layer and the output layer; $k_{2}$, the bias term of the output layer; and $M\left(x, F, k_{1}\right)$, the output of hidden layer neurons. $\hat{F}, \hat{W}, \hat{k_{1}}$, and $\hat{k_{2}}$ are the optimized parameters of the MLP.

As is typical for the MLPs trained to estimate posterior probabilities, all the MLPs are trained using teaching signal $\hat{y}$, which is " 1.0 " for the monophone associated with the central frame and " 0 " for all the others. We use the standard error back-propagation algorithm to optimize $\hat{F}, \hat{W}, \hat{k_{1}}$, and $\hat{k_{2}}$.

During the application phase, the output layer of the MLP is removed. Therefore, the output of MLP-OL is defined by $M\left(x, \hat{F}, \hat{k_{1}}\right) . M\left(x, \hat{F}, \hat{k_{1}}\right)$ can be interpreted as the convolution of $x$ and the elements of $F$ with a nonlinear sigmoid function. Therefore, $M\left(x, \hat{F}, \hat{k_{1}}\right)$ has a fixed frequency response that can improve the distinguishability of $x$. The filter constructed using the above procedure is called a "matched filter."

\subsection{Tandem Approach of Acoustic Model}

To recognize the output of matched filters, the HMM/MLP tandem approach is used in HATS [16]. In this approach, the input feature vector is classified under monophones by the MLP, and the output vector of the MLP is decoded using Gaussian mixture hidden Markov models, which use the output vector of MLPs as the observed feature vector sequence.

The MLPs in the tandem approach are also trained to estimate the posterior probabilities of the associated monophones; the teaching signal for training is " 1.0 " for the monophone associated with the central frame and "0" for all the others.

Because output vectors of the MLPs have a skew symmetry and correlations between their dimensions, they are

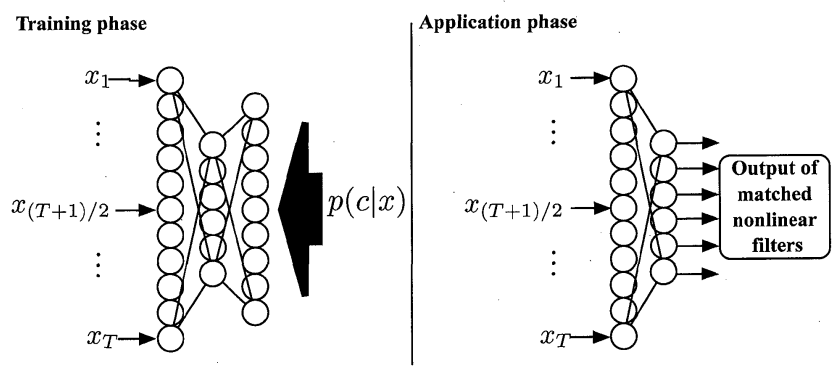

Fig. 3 Diagram of MLP-OL. incompatible with the Gaussian mixture hidden Markov models. Therefore, it is necessary to adapt the feature distribution to the Gaussian model in order to achieve stable recognition. Typically, logarithmic function and KarhunenLoeve transformation (KLT) are used for symmetrizing and diagonalizing, respectively.

\section{Amplitude and Frequency Modulation Classifiers (AFMC)}

In this section, we describe our system, which is called the "amplitude and frequency modulation combination" (AFMC), that employs AM and FM for speech.

Figure 4 shows the block diagram of our automatic speech recognition (ASR) system.

\subsection{Filter Bank}

First, a filter bank that is designed to simulate the frequency responses of the basal membrane of the lining of the inner ear is used. The frequency responses of the filter bank are the same as those of the filter bank in the PLP analysis [1]. Unlike PLP and TRAPS-like classifiers [12], [13], this filter bank is implemented using FIR filters in order to preserve the carrier information.

The frequency response is defined by the following equations:

$$
\begin{aligned}
& \Omega(\omega)=6 \log \left(\frac{\omega}{1200 \pi}+\sqrt{\left(\frac{\omega}{1200 \pi}\right)^{2}+1}\right), \\
& \Psi(z)= \begin{cases}0 & z<-1.3, \\
10^{2.5(z+0.5)} & -1.3 \leq z \leq-0.5, \\
1 & -0.5<z<0.5, \\
10^{-1.0(z-0.5)} & 0.5 \leq z \leq 2.5, \\
0 & 2.5<z\end{cases}
\end{aligned}
$$

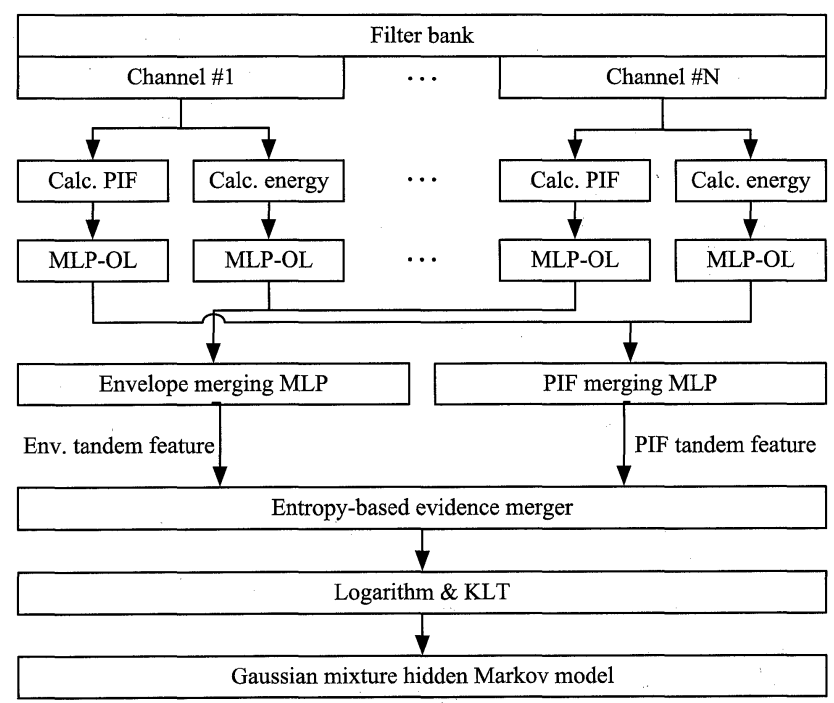

Fig. 4 Block diagram of our proposed system: The details of the filter bank are described in Sect. 4.1, MLP-OLs are described in Sect. 4.2, merging MLPs are described in Sect. 4.3, and the entropy-based evidence merger is described in Sect. 4.4. 


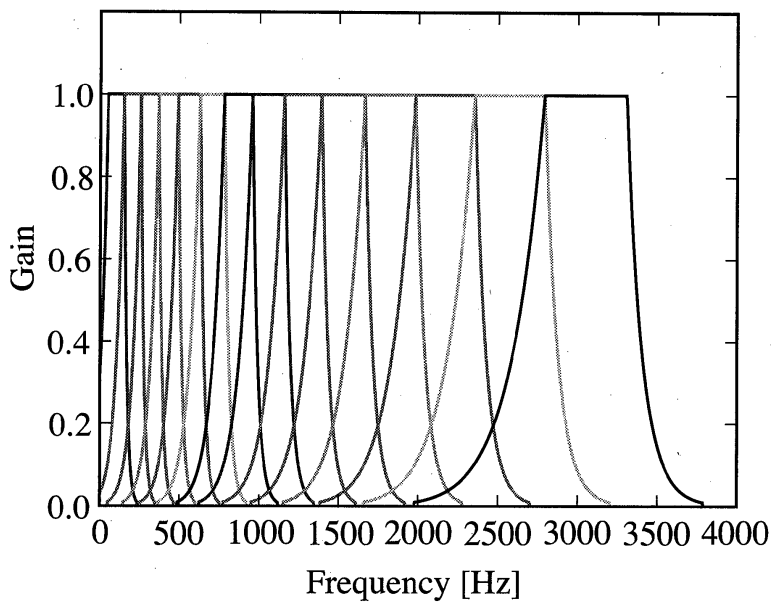

Fig. 5 Frequency response of filter $G_{b}(\omega)$.

$$
G_{b}(\omega)=\Psi(\Omega(\omega)-n) .
$$

Here, $\Omega$ is the mapping from the angular speed to the bark frequency; $\Psi(z)$, the shape of the band-pass filter (BPF) that simulates the frequency masking pattern of human auditory perception; and $G_{b}(\omega)$, the frequency response of the $b^{\text {th }}$ filter. $G_{b}(\omega)$ is depicted in Fig. 5 .

Subsequently, we calculate the logarithmic envelope $E_{b}(n)$ and logarithmic PIF $P_{b}(n)$ by the following expressions.

$$
\begin{aligned}
& E_{b}(n)=\log \left(\left|x_{b}(n)\right|^{2}\right) . \\
& P_{b}(n)=\log \left(P I F\left(x_{b}(n)\right)\right) .
\end{aligned}
$$

Here, $x_{b}(n)$ is the output of the $b^{\text {th }}$ channel of the filter bank, and $\operatorname{PIF}\left(x_{b}(n)\right)$ is the PIF of $x_{b}(n)$ at time $n$.

We then apply a low-pass filter to $E_{b}(n)$ and $P_{b}(n)$ in order to resample them. We resampled the low-pass filtered $E_{b}(n)$ and $P_{b}(n)$ at $100 \mathrm{~Hz}$.

Figure 6 depicts the $E_{b}(n)$ and $P_{b}(n)$.

\subsection{Matched Modulation Filter}

The resampled $P_{b}(n)$ and the resampled $E_{b}(n)$ of the $b^{\text {th }}$ sub-band signal are processed by the MLP-OLs described in Sect. 3.1.

The input signal $x_{i}$ of the $i^{\text {th }}$ neuron in the input layer of the MLP-OL at time $n$ is defined by

$$
x_{i}=E_{b}\left(n+i-\frac{L+1}{2}\right),
$$

for the envelope analyzer, and

$$
x_{i}=P_{b}\left(n+i-\frac{L+1}{2}\right),
$$

for the PIF analyzer.

Here, $L$ is the number of dimensions of the input vector (must be odd); $E_{b}(n)$, the $n^{\text {th }}$ sample of the resampled logarithmic energy of the $b^{\text {th }}$ channel; and $P_{b}(n)$, the $n^{\text {th }}$ sample of the resampled PIF of the $b^{\text {th }}$ channel. In this study, we assume $L=51$.

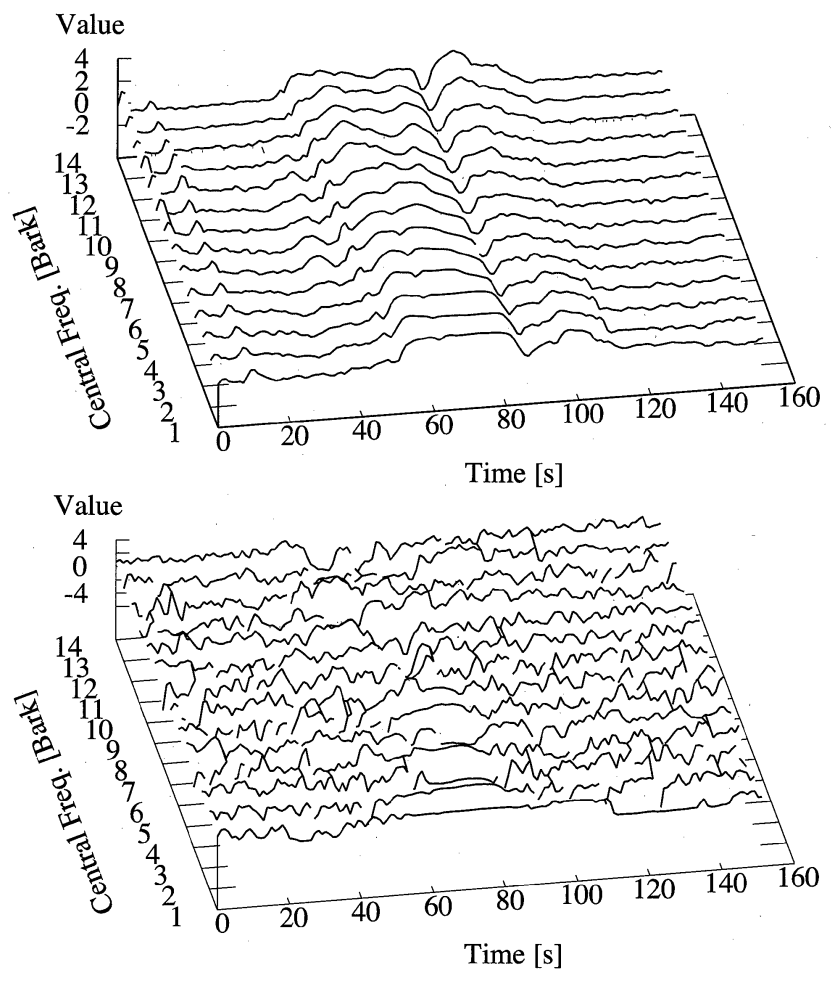

Fig. 6 Resampled $E_{b}(n)$ (top) and resampled $P_{b}(n)$ (bottom) of speech: (The mean and variance are normalized to 0.5 and 0.25 , respectively.)

\subsection{Tandem Approach}

To recognize the output of the matched filters, we use the HMM/MLP tandem approach employed in acoustic modeling and described in Sect. 3.2.

Because AM and FM might have different robustness for environmental effects, merging them adaptively is an effective way for acquiring robustness. Therefore, we merge estimates from two MLPs; envelope (AM) merging MLP and PIF (FM) merging MLP, adaptively.

\subsection{Entropy-Based Evidence Merger}

Now, we have observed two streams of the MLP output score sequence by the procedure described in Sect. 4.3. We merge them before using them as features of GM-HMMs.

We used the entropy-based combination of tandem acoustic models, which has been introduced by Ikbal et al. [17].

First, we calculated the approximate posterior probability for the $m^{\text {th }} \operatorname{MLP} p\left(c \mid x^{m}\right)$ with the expression

$$
p\left(c \mid x^{m}\right)=\frac{\left(\exp \left(-y_{i(c)}^{m}\right)-1\right)^{-1}}{\sum_{d \in C}\left(\exp \left(-y_{i(d)}^{m}\right)-1\right)^{-1}} .
$$

Here, $x^{m}$ is the input vector of the $m^{\text {th }}$ MLP; $y^{m}$, the output vector of the $m^{\text {th }}$ MLP; $C$, the set of target classes (in this study, $C$ is the set of monophones); and $i(c)$, the mapping from the elements of $C$ to the dimension index of $y^{m}$. 
This equation implies the cancellation of the sigmoid function and the application of the soft-max transfer function to the output of the MLP.

In concrete terms, $x^{1}$ is the input vector of the envelope-merging MLP; $x^{2}$, the input vector of the PIFmerging MLP; $y^{1}$, the output vector of the envelope-merging MLP; and $y^{2}$, the output vector of the PIF-merging MLP.

The conditional entropy of $x^{m}$ is estimated as

$$
H^{m}\left(C \mid x^{m}\right)=\sum_{c \in C}-p\left(c \mid x^{m}\right) \log p\left(c \mid x^{m}\right) .
$$

The weights of $y^{m}$ are determined by taking the inverse of the conditional entropy $h^{m}$ :

$$
w^{m}=\frac{\left\{H^{m}\left(C \mid x^{m}\right)\right\}^{-1}}{\sum_{j=1}^{M}\left\{H^{j}\left(C \mid x^{j}\right)\right\}^{-1}},
$$

where $M$ is the number of MLPs (in this study, $M$ is 2).

Finally, we obtain the merged output $\hat{y}$ from the expression

$$
\hat{y}_{i}=\sum_{m=1}^{M} w^{m} \log \left(y_{i}^{m}\right) .
$$

Because the dimensions of $\hat{y}$ correlate with each other, $\hat{y}$ is incompatible with the diagonal GMMs.

It is necessary to transform $\hat{y}$ for dimensionality reduction and decorrelation. For this, we use the KLT.

\section{Experimental Setup}

In this section, we evaluate the performance of the proposed system by conducting experiments.

We performed continuous digit recognition of reverberant speech in an experiment. The training set used for both the MLP and HMM comprised 8,440 utterances from 110 speakers, and it was taken from CENSREC-1 [18]. Every test set that was used for both the clean environment test and reverberant environment tests comprised 2002 utterances from 104 speakers. The sampling rate for all the input signals was fixed at $8000 \mathrm{~Hz}$; hence, the number of filter bank channels was 14 .

We prepared four impulse responses for producing reverberant speech that simulates the reverb at the following locations:

- Room (small reverberant room)

- Meeting room

- Silo

- Theater

- Cathedral

Figure 7 shows the time characteristics of the reverb calculated by using the expression

$$
R(n)=10 \log _{10}\left(\frac{\sum_{m=n}^{\infty}\{h(m)\}^{2}}{\sum_{m=0}^{\infty}\{h(m)\}^{2}}\right),
$$

where $h(m)$ is the impulse response of the reverb.

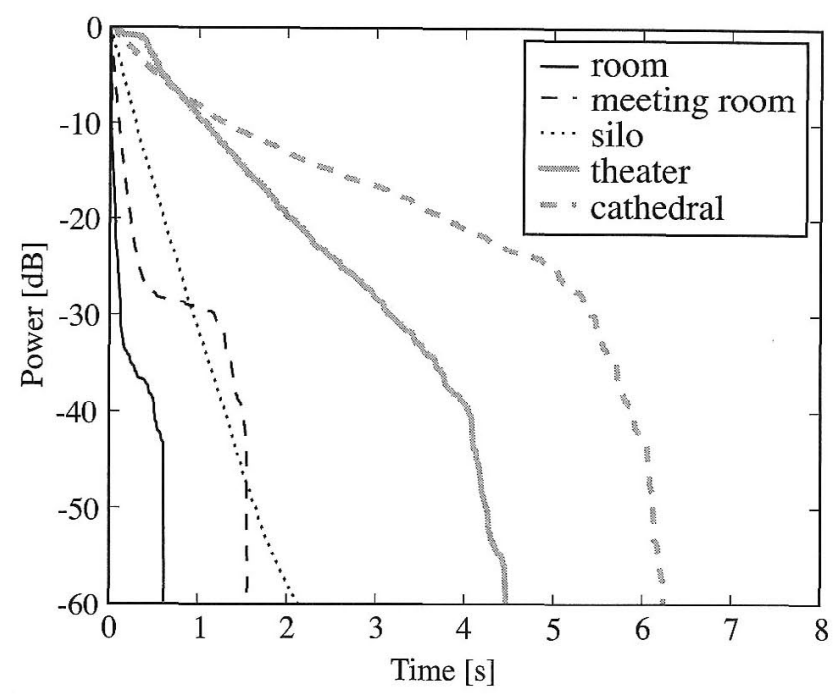

Fig. 7 Time characteristics of reverb.

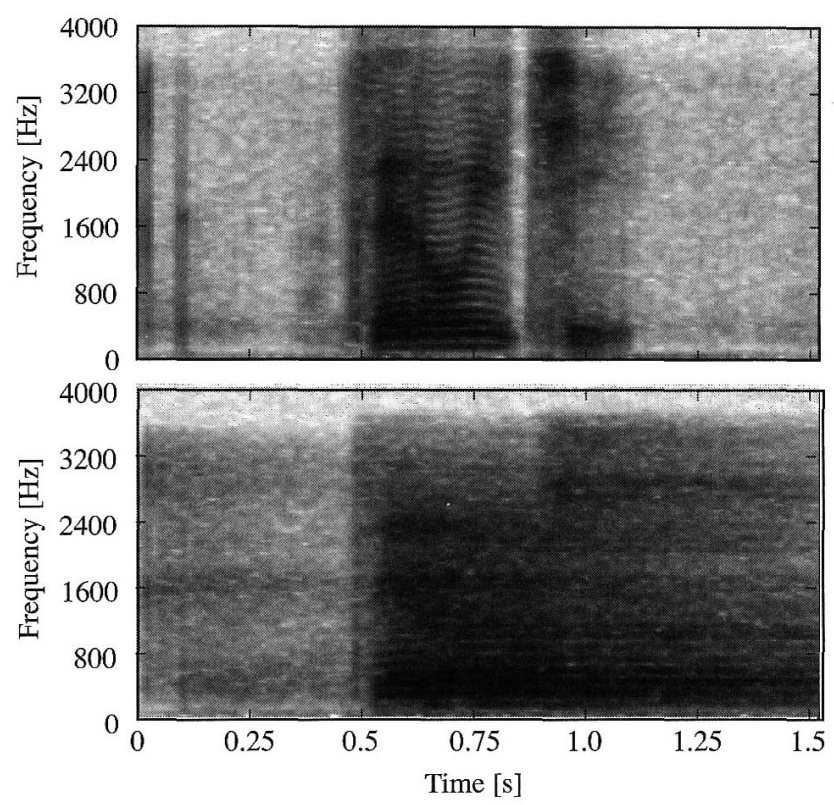

Fig. 8 Spectrograms for clean speech (top) and reverberant speech in silo (bottom).

The reverberation test set was generated by convoluting the impulse responses to signals in clean environments. Figure 8 shows the spectrograms for clean speech and reproduced reverberant speech using the impulse response of the silo (the amplitude at corresponds time and frequency is represented as the intensity.)

The baseline is the MFCC and energy feature extraction system that is augmented by the derivation and acceleration of the MFCC and energy. (MFCC_E_D_A; 39 dims.)

We compared several methods, as described below.

- AIF

A recognizer based on AIF features that are defined by removing the ALE features from the AIF/ALE [7]. (augmented by its derivations and accelerations; 42 
dims.)

- $\mathrm{AM}$

A recognizer based on AM features was constructed by removing the PIF MLPs from the AFMC. This method is equivalent to HATS [12], [13].

- FM

A recognizer based on FM features was constructed by removing the envelope MLPs from the AFMC.

- AFMC

The proposed method.

All the HMMs and MLPs are trained to be independent of the gender and speaker.

Table 1 shows the word accuracy $(100-\%$ insertion \%deletion - \%substitution) of the compared methods in the test set.

\section{Discussions}

From Table 1, it is observed that the proposed system exhibits considerable improvement as compared to other methods.

Our FM method exhibits considerable improvement copared with AIF. The effectiveness of the method which capture temporal structures in IFs using MLP-OL is confirmed by the experiment.

In comparison with AM, our system reduced $8.83 \%$ of the word errors in reverberant environments. This shows the advantage of the FM analysis employing the PIF in reverberant speech recognition.

We observed that the FM method has low accuracy as compared with the AM method. However, the FM information of the signal appears to be significant since the combination method (AFMC) achieved an accuracy higher than that of AM method.

The essence of the FM analysis is discussed in the following section.

\subsection{Environmental Adaptation}

The average weights allocated to streams that are determined by the entropy-based evidence merger are presented in Table 2. The table shows that the AM information is dominant in the clean environment; however, the weights in the FM analysis increase in reverberant environments.

Figure 9 shows the time trajectory of the weights determined by the entropy-based evidence merger.

Table 1 Word accuracies of compared methods as percentages.

\begin{tabular}{l|rrrrr}
\hline & MFCC & AIF & AM & FM & AFMC \\
\hline Clean & 97.88 & 89.75 & 97.47 & 90.86 & 98.40 \\
Room & 78.93 & 37.15 & 86.64 & 72.20 & 92.08 \\
Meeting room & 34.35 & 27.68 & 41.81 & 44.55 & 52.03 \\
Silo & 26.21 & 13.51 & 25.76 & 26.43 & 31.44 \\
Theater & 23.11 & 14.07 & 31.18 & 21.43 & 34.99 \\
Cathedral & 15.83 & 1.25 & 16.83 & 13.72 & 17.88 \\
\hline Avg. reverb & 35.69 & 18.73 & 40.44 & 35.67 & 45.68 \\
\hline
\end{tabular}

In clean environments, the AM information is dominant most of the time. However, in reverberant environments, the AM information is degraded by reverberations and the FM information is relatively dominant at the end of the signal.

\subsection{Measuring the Information in FM}

In order to measure the information in FM, we calculate the mutual entropy of the vector-quantized FM patterns.

First, we define partial specific mutual entropy $I_{c}(X)$ as

$$
\begin{aligned}
I_{c}(X)= & -\sum_{x \in X} p(x \mid c) \log p(x \mid c), \\
& =H(X)-H(X \mid c) .
\end{aligned}
$$

Here, $H(X)$ is the entropy of $X$ and $H(X \mid C)$ is the conditional entropy of $X$.

$I_{c}(X)$ represents the locality about $x$ in feature space $X$ when $C$ is fixed at $c$.

We then derive the mutual entropy as the expectation of $I_{c}(X)$.

$$
I(X ; C)=\sum_{c \in C} p(c) I_{c}(X) .
$$

The mutual entropy $I(X ; C)$ represents the expectation of the locality about $x$ in feature space $X$ when $C$ is fixed. A

Table 2 Average weights of AM and FM information in the environments considered in the entropy-based evidence merger.

\begin{tabular}{l|rr}
\hline & AM & FM \\
\hline clean & 81.35 & 18.65 \\
room & 76.31 & 23.69 \\
meeting room & 61.76 & 38.24 \\
theater & 50.26 & 49.74 \\
silo & 55.85 & 44.15 \\
cathedral & 47.75 & 52.25 \\
\hline
\end{tabular}

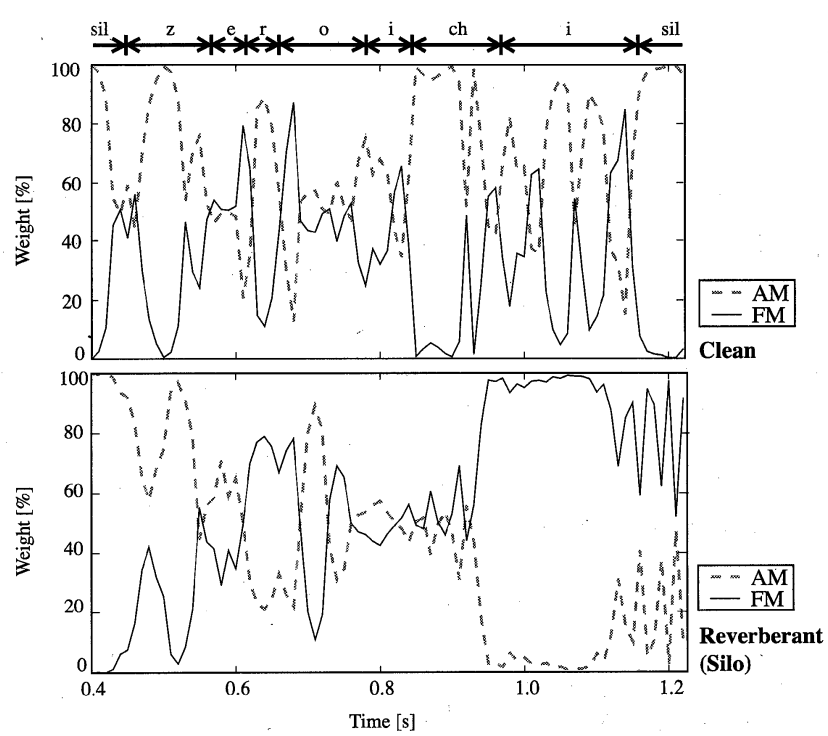

Fig. 9 Time trajectory of weighting factor. (top: clean, bottom: silo) 
Table $3 I(x ; c)$ for the AM feature vector and FM feature vector of the $5^{\text {th }}$ sub-band.

\begin{tabular}{r|rr|rr}
\hline & \multicolumn{2}{|c|}{ AM } & \multicolumn{2}{c}{ FM } \\
$I_{c}(X)$ & Clean & Reverb & Clean & Reverb \\
\hline $\mathrm{a}$ & 0.62 & 0.73 & 0.17 & 0.16 \\
$\mathrm{i}$ & 0.77 & 0.94 & 0.24 & 0.37 \\
$\mathrm{u}$ & 0.93 & 1.10 & 0.29 & 0.49 \\
$\mathrm{e}$ & 1.80 & 1.49 & 0.38 & 0.28 \\
$\mathrm{o}$ & 1.11 & 1.13 & 0.10 & 0.34 \\
$\mathrm{~N}$ & 0.87 & 0.99 & 0.16 & 0.29 \\
$\mathrm{ch}$ & 1.51 & 0.93 & 0.40 & 0.15 \\
$\mathrm{~g}$ & 1.44 & 1.28 & 0.17 & 0.19 \\
$\mathrm{~h}$ & 2.23 & 0.89 & 0.66 & 0.32 \\
$\mathrm{k}$ & 2.10 & 1.08 & 0.43 & 0.28 \\
$\mathrm{ky}$ & 1.77 & 0.81 & 0.58 & 0.18 \\
$\mathrm{~m}$ & 1.48 & 1.20 & 0.86 & 0.43 \\
$\mathrm{n}$ & 1.02 & 0.75 & 0.29 & 0.05 \\
$\mathrm{r}$ & 1.45 & 1.02 & 0.32 & 0.15 \\
$\mathrm{~s}$ & 1.42 & 0.72 & 0.50 & 0.31 \\
$\mathrm{y}$ & 1.34 & 1.10 & 0.51 & 0.34 \\
$\mathrm{z}$ & 2.21 & 1.59 & 0.51 & 0.28 \\
$\mathrm{sp}$ & 1.78 & 1.70 & 0.44 & 0.30 \\
\hline \hline$I(X ; C)$ & 1.30 & 1.15 & 0.33 & 0.29 \\
\hline
\end{tabular}

decrease in $I(X ; C)$ represents the degradation of the locality of feature in the feature space. The locality of the feature will be an identification factor. low.

The data sets we used for the evaluation are listed be-

- Clean

The data set is identical to the "clean" data set defined in Sect. 5.

- Reverb

The data set contains all the feature vectors in "room," "meeting room," "silo," "theater," and "cathedral," which were defined in Sect. 5 .

$I_{c}(X)$ and $I(X ; C)$ for the AM and FM feature vectors of the $5^{\text {th }}$ sub-band are depicted in Table 3.

The accuracy of discrimination depends on the distributional overlap between the target classes. Therefore, the entropy value of the feature vector does not affect the recognition accuracy. However, a decrease in $I(X ; C)$ causes a degradation in the feature vector because the degradation of the locality in feature space will result in a distributional overlap.

Figure 10 depicts the differences in $I(X ; C)$ between the clean and the reverb environments for all the sub-bands.

In the AM feature vector, decreases in the partial specific mutual entropies are observed in many monophones and all bands. However, in the FM feature vector, partial specific mutual entropies are stable at all monophones in most of the bands. Therefore, It is considered that FM analysis is important for reverberant speech recognition.

\subsection{Frequency Responses of Matched Filters}

The average frequency responses of the AM-matched filter and FM-matched filter are depicted in Fig. 11.

The effectiveness of applying a BPF for envelope mod-

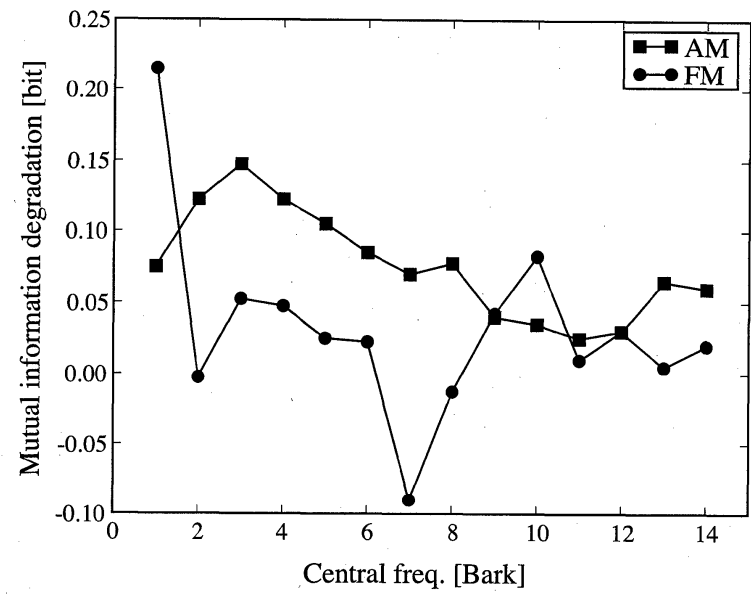

Fig. 10 Differences between mutual information $I(X ; C)$ of clean and reverb environments.
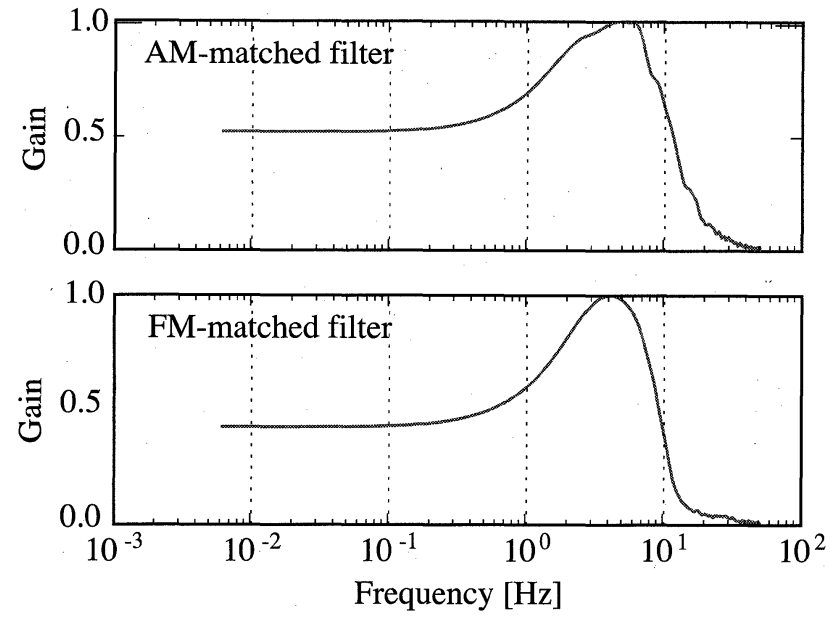

Fig. 11 Average frequency response of AM-matched filters (top) and average frequency response of FM-matched filters (bottom).

ulation is well known and been employed in many methods [5], [10]. AM-matched filters are also constructed as BPFs that emphasize envelope modulation around a frequency of $4 \mathrm{~Hz}$. The behavior is similar to the human auditory perception of AM [19], [20].

Interestingly, the frequency response pattern of FMmatched filters has a similarity to that of AM matched filters. The phonetic information in the carrier signal is distributed around a frequency of $4 \mathrm{~Hz}$ in $\mathrm{FM}$.

\section{Conclusion}

In this paper, we defined the PIF of a signal, which is useful for pattern recognition in carrier signals.

We proposed a method based on a tandem approach to analyze the envelope and PIF of narrowband signals; the method is called AFMC, and it comprises AM analysis MLPs, FM analysis MLPs, and an entropy-based evidence merger.

We evaluated the performance of the proposed method 
by performing continuous digit recognition of reverberant speech. The proposed system exhibited considerable improvement with regard to the MFCC feature extraction system.

Further, the essence of FM is investigated. In contrast with AM information, the FM pattern does not lose its information in reverberant environments. The importance of FM analysis is significant for environments that involve reverb. It was observed that the FM rate that is available for ASR is limited to around $4 \mathrm{~Hz}$.

\section{Acknowledgements}

This study was supported by the Advanced Research Institute for Science and Engineering at Waseda University under the project "Research on Multi-Modal Human Interface Aiming for Spontaneous Communication System." It was partially supported by Grant-in-Aid for Scientific Research (B) $(17300066,2007)$ from the Ministry of Education, Science, Sports and Culture, Japan.

The present study was conducted using the impulse response and speech data with microphone array data of the RWCP Sound Scene Database in Real Acoustic Environment.

\section{References}

[1] H. Hermansky, "Perceptual linear predictive (PLP) analysis of speech," J. Acousti. Soc. Am., vol.87, pp.1738-1752, April 1990.

[2] H. Hermansky and S. Sharma, "TRAPS - Classifiers of temporal patterns,” Proc. ICSLP'98, Sydney, Australia, Nov. 1998.

[3] K. Yoshida, M. Kazama, and M. Tohyama, "Pitch and speech-rate conversion using envelope modulation modeling," Proc. ICASSP2002, I.435-I.428, Orland, 2002.

[4] H. Hermansky, "Should recognizers have ears?," Speech Commun., vol.25, no.1-3, pp.3-27, 1998.

[5] H. Hermansky and N. Morgan, "RASTA processing of speech," IEEE Trans. Speech Audio Process., vol.2, no.4, pp.578-589, Oct. 1994.

[6] T. Houtgast and H.J.M. Steeneken, "A review of the MTF concept in room acoustics and its use for estimating speech intelligibility in auditoria," J. Acoust. Soc. Am., vol.77, pp.1069-1077, March 1985.

[7] Y. Wang, J. Hansen, G.K. Allu, and R. Kumaresan, "Average Instantaneous Frequency (AIF) and Average Log-Envelopes (ALE) for ASR with the aurora 2 database," Proc. Eurospeech 2003, pp.25-28, 2003.

[8] D. Dimitriadis, P. Maragos, and A. Potamianos, "Robust AM-FM features for speech recognition," IEEE Signal Process. Lett., vol.12, no.9, pp.621-624, Sept. 2005.

[9] J. Bilmes, "Maximum mutual information based reduction strategies for cross correlation based joint distributional modeling," Proc. ICASSP-98, pp.469-472, Seattle, May 1998.

[10] N. Kanedera, T. Arai, and T. Funada, "Robust automatic speech recognition emphasizing important modulation spectrum," IEICE Trans. Inf. \& Syst. (Japanese Edition), vol.J84-D-II, no.7, pp.12611269, July 2001.

[11] N. Morgan, Q. Zhu, A. Stolcke, K. Sönmez, S. Sivadas, T. Shinozaki, M. Ostendorf, P. Jain, H. Hermansky, D. Ellis, G. Doddington, B. Chen, Ö. Çetin, H. Bourlard, and M. Athineos, "Pushing the envelope - Aside," IEEE Signal Process. Mag., vol.22, no.5, pp.81-88, Sept. 2005.

[12] B. Chen, S. Chang, and S. Sivadas, "Learning discriminative tem- poral patterns in speech: Development of novel TRAPS-like classifiers," Proc. Eurospeech, pp.429-432, Geneve, 2003.

[13] B. Chen, S. Chang, and S. Sivadas, "Learning long term temporal features in LVCSR using neural networks," Proc. INTERSPEECHICSLP-2004, pp.612-615, Jeju Island, Korea, Oct. 2004.

[14] S. Okawa, E. Bocchieri, and A. Potamianos, "Multi-band speech recognition in noisy environments," Proc. ICASSP-98, pp.641-644, Seattle, Washington, USA, May 1998.

[15] H. Suzuki, F. Ma, H. Izumi, O. Yamazaki, S. Okawa, and K. Kido, "Instantaneous frequencies of signals obtained by the analytic signal," Acoustical Science \& Technology, vol.27, no.3, pp.163-170, May 2006.

[16] H. Hermansky, D.P.W. Ellis, and S. Sharma, "Tandem connectionist feature extraction for conventional HMM systems," Proc. ICASSP2000, pp.1635-1638, Istanbul, June 2000.

[17] S. Ikbal, H. Misra, S. Sivadas, H. Hermansky, and H. Bourlard, "Entropy based combination of tandem representations for noise robust ASR," Proc. INTERSPEECH-ICSLP-2004, pp.2553-2556, Jeju Island, Korea, Oct. 2004.

[18] CENSREC-1: http://sp.shinshu-u.ac.jp/CENSREC/ja/CENSREC/AURORA-2J/

[19] E. Zwicker, "Die Grenzen der Hoerbarkeit der Amplitudenmodulation under der Frequenzmodulation eines Tones," Acusstica, vol.2, pp.125-133, 1952.

[20] G. Green, Temporal Aspects of Audition, Ph.D. Thesis, Oxford University, 1976.

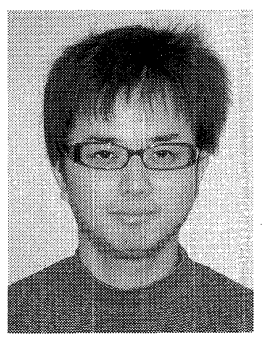

Yotaro Kubo received his B.E. degree in Information and Computer Science from Waseda University, Tokyo, Japan, in 2007, and he is working for the M. Eng. (and Dr. Eng.) degree. His research interests include machine learning, pattern recognition, and signal processing.

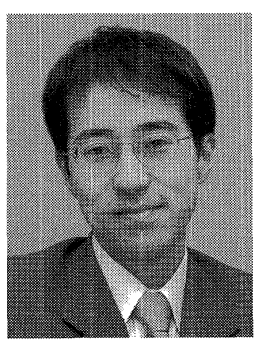

Shigeki Okawa received his B. Eng., M. Eng., and Dr. Eng. degrees from Waseda University, Tokyo, Japan, in 1992, 1994, and 1996, respectively (all in electrical engineering). He was a Research Fellow of the Japan Society for the Promotion of Science from 1994 to 1998 . During the period (1996 to 1997), he joined AT\&T Labs, New Jersey, USA. From 1998 to 2006, he was an Associate Professor at the Dept. of Network Science, Chiba Institute of Technology, Narashino, Japan. Since 2006, he has been an Associate Professor at the Dept. of Advanced Robotics, Chiba Institute of Technology. His research interests include spoken language processing, signal processing, and human-computer interaction. He is a member of IEEE, IPSJ, JSAI, ASJ, RSJ, and other organizations. 


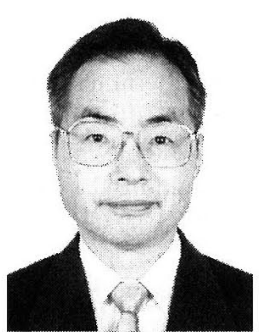

Akira Kurematsu received his B.E. degree in Electrical Communication from Waseda University in 1961. He received a Ph.D. degrce from Waseda University in 1971. In 1961, he joined the Research and Development Laboratories of $\mathrm{KDD}$ where he was engaged in research on pattern recognition, speech signal processing, and human communication systems. In 1983, he was appointed Deputy Director of KDD R\&D Labs. From 1986 to 1993, he was the president of the ATR Interpreting Telephony Research Laboratories. He was a professor at the Department of Electronic Engineering, University of Electro-Communications, from 1993 to 2004. Since 2004, he has been a visiting professor at Waseda University.

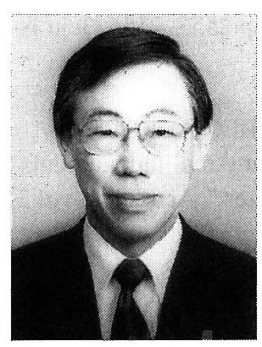

Katsuhiko Shirai received his B. Eng., M. Eng., and Dr. Eng. degrees from Waseda University, Tokyo, Japan, in 1963, 1965, and 1973, respectively (all in Electrical Engineering). Since 1991, he has been a professor at the Department of Information and Computer Science, Waseda University. His research interests include spoken language processing and high-level synthesis of ASIC and CAI systems. He is a member of the Information Processing Society of Japan, Japanese Society of Artificial Intelligence, and the Institute of Electrical and Electronics Engineers in addition to other organizations. 